\title{
Watch your catch. Two cases of effective mechanical thrombectomy in large cerebral vessel occlusion by tumor fragments
}

Christian Hendrik Claassen*1, Lars Udo Krause ${ }^{1}$, Stephan Lowens², Evgeny Minin³, Jan Rothaupt2 1Department of Neurology, ${ }^{2}$ Department of Radiology and ${ }^{3}$ Institute of Pathology, Klinikum Osnabrück, Germany

\section{Background}

Over the last years, mechanical thrombectomy has become a common method of treatment for ischemic stroke caused by large cerebral vessel occlusion. With this technique it is possible to histologically examine cerebral thromboembolic material ${ }^{1}$. Most cerebral emboli contain platelets, red blood cells, fibrin and von Willebrand factor ${ }^{2}$. However, there is evidence that histological and molecular analysis can help to distinguish cardiogenic from arteriogenic clots and thus help to identify stroke etiology 3,4 . Here we report two cases of effective mechanical thrombectomy in which large cerebral vessel occlusion by tumor fragments was the initial manifestation of neoplastic disease.

\section{Case 1}

A 73-year-old man was presented to our emergency department with wake-up right-side hemiparesis and aphasia (NIHSS 10). Pre-existing conditions included stented infrarenal abdominal aortic aneurysm and anticoagulation with Phenprocoumon due to repeated thrombosis of a femoro-femoral bypass. Multimodal CT revealed left MCA occlusion and a large perfusion mismatch. Transaxillary mechanical thrombectomy was performed. Histological examination of the macroscopically atypical embolus showed tissue consistent with papillary fibroelastoma, a very rare benign cardiac tumor. Transesophageal ultrasound showed a $9^{*} 7 \mathrm{~mm}$ mass adjacent to the leftcoronary aortic valve cusp. The patient underwent aortic valve replacement and recovered without relevant neurological deficits.

\section{Case 2}

A 45-year-old woman was admitted to our emergency department with acute onset right-side hemiparesis and aphasia (NIHSS 17). Multimodal CT showed left MCA and ACA occlusion and a left thoracic tumorous lesion of $9^{*} 12^{*} 13 \mathrm{~cm}$. Mechanical thrombectomy of occlusions of left M1 and A. pericallosa retrieved emboli that appeared atypical macroscopically. Immunohistochemical analysis confirmed tissue of an aggressive angio-invasive lung carcinoma. The patient underwent left upper lung lobectomy and received adjuvant chemoradiotherapy. At discharge mild aphasia and mild right-side hemiataxia remained.

\section{Discussion}

These two cases show that careful macroscopic examination and selective histopathologic analysis of specimens retrieved in mechanical thrombectomy may help to identify rare causes of stroke. In both cases, embolism of tumor fragments resulted in embolic stroke. Immediate etiologic classification helped to allocate specific treatment. In the future, more routine macroscopic, histological or maybe even molecular examination of MT specimens might help reduce the amount of ESUS patients.

1. Marder VJ, Chute DJ, Starkman S, Abolian AM, Kidwell C, Liebeskind D, Ovbiagele B, Vinuela F, Duckwiler G, Jahan R, et al. (2006): Analysis of thrombi retrieved from cerebral arteries of patients with acute ischemic stroke. Stroke 37, 2086-2093

2. Krajíčková D, Krajina A, Šteiner I, Vyšata O, Herzig R, Lojík M, Chovanec V, Raupach J, Renc O, Waishaupt J, et al. (2018): Fibrin Clot Architecture in Acute Ischemic Stroke Treated With Mechanical Thrombectomy With Stent-Retrievers Cohort Study. Circ J 82, 866-873

3. Ahn SH, Hong R, Choo IS, Heo JH, Nam HS, Kang HG, Kim HW, Kim JH (2016): Histologic features of acute thrombi retrieved from stroke patients during mechanical reperfusion therapy. International Journal of Stroke 11, 1036-1044

4. Baek BH, Kim HS, Yoon W, Lee YY, Baek JM, Kim EH, Kim SK (2018): Inflammatory mediator expression within retrieved clots in acute ischemic stroke. Ann Clin Transl Neurol $\underline{5}, 273-279$

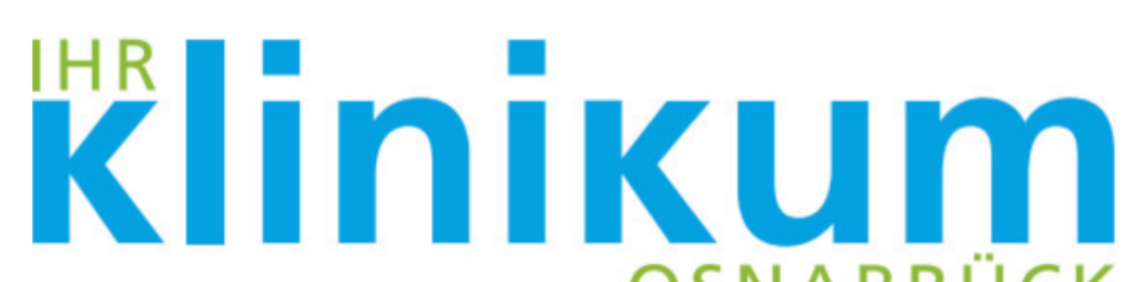

OSNABRÜCK 